\title{
TENDENCIAS ACTUALES EN LA FORMACIÓN DEL PROFESORADO DE CIENCIAS*
}

\author{
FURIÓ MAS, C.J. \\ Departament de Didàctica de les Ciències Experimentals i Socials. Universitat de València.
}

\section{SUMMARY}

In the last few years, there has been a significant growth of interest in science teacher education. An analysis of recent science teacher education research is offered in this paper. This study a:sswers the major question: What does research tell us about the recent trends in teacher preparation? We are beginning to understand that in the in-service or preservice training is it very important to know the effects of: $a$ ) a limited content knowledge; $b$ ) the «commonsense» conceptions, behaviors and attitudes about different aspects of science teaching; c) the need of integration «knowledge-practice» in programs of teacher education and that; $d$ ) theses obstacles to didactic knowledge adquisition alone will be overcome when the teachers are placed in a situation which explicitly demands a collective reflection through the research.

\section{INTRODUCCIÓN}

Durante las dos últimas décadas, la investigación en didáctica de las ciencias ha focalizado su atención en el aprendizaje y, en particular, se ha caracterizado por haber estudiado en profundidad el conocimiento anterior de los estudiantes en muy diversos dominios de las ciencias. Estos resultados han puesto en cuestión el paradigma de enseñanza-aprendizaje de las ciencias basado en la transmisión verbal del conocimiento científico acabado y están propugnando su sustitución por el paradigma emergente de orientación constructivista (Gil 1983, Driver 1988, Novak 1988). No obstante, la práctica docente está todavía muy lejos de asumir este cambio paradigmático (Gil 1994). Al propio tiempo, estamos asistiendo a una incipiente consolidación de la Didáctica de las Ciencias como cuerpo propio de conocimientos teóricos que no sólo está incorporando bien los avances de la psicología cognitiva y de las ciencias de la educación respecto al aprendizaje (Tiberghien 1985, Gutiérrez 1987, Aliberas et al. 1989), sino que además está llamado a desempeñar el papel de núcleo vertebrador de aquellos conocimientos con los logros de la psicología afectiva (Pintrich et al. 1993) y los de la historia y epistemología de las Ciencias (Furió y Gil 1989, Matthews 1990, Duschl y Gitomer 1991).

Ahora bien, el énfasis inicialmente puesto por la didáctica de las ciencias en diferenciar el aprendizaje de la enseñanza y en atribuir, desde planteamientos construc- tivistas, la responsabilidad del aprendizaje al propio aprendiz, está derivando desde hace unos pocos años -y cada vez con mayor interés- hacia el estudio de la enseñanzć. $y$, en particular, hacia el de su principal protagonista: el profesorado. En nuestra opinión, es a partir de la mitad de la década de los $80 \mathrm{y}$, en particular, desde el II Simposium sobre errores conceptuales en ciencias y en matemáticas celebrado en Cornell University (1987), cuando la comunidad cientifica en este dominio de la investigación está dirigiendo sus recursos y esfuerzos hacia el tema de la formación del profesoracio. Este interés no es meramente especulativo, pues no en balde las administraciones se han dado cuenta de la impcrtancia que tiene la figura del profesor como mediador del proceso en cualquier reforma de las enseñanzas y de cómı la propia investigación está poniendo de relieve las diferencias encontradas entre los objetivos eđucativos diseñados por los planificadores del currículo y los que os profesores llevan realmente a la práctica (Cronin Jones 1991). Es decir, se está pasando de investigar to que piensa y hace el alumno en clase hacia lo que piensa y hace el profesor, tratando de analizar su actividad y así poder descifrar las claves de su desarrollo profesional. En particular, habrá que determinar cuáles son sus necesidades formativas y qué factores pueden influir en una continua preparación para el cambio didáctico (Gil 1991). 
Por otra parte, estas orientaciones de la investigación diđáctica en la formación del profesorado de ciencias, están siendo convergentes con las críticas al paradigma de investigación sobre formación del profesorado basado en la psicología asociacionista que se vienen realizando desde el campo đe las ciencias de la educación. En particular, se critica su enfoque proceso-producto y su exclusiva preocupación por la búsqueda y tipificación de comportamientos en profesores excelentes con el fín de simularlos en cursos y adiestrar a los futuros profesionales de la educación en aquellas conductas eficaces (Gimeno 1990). Seolvida que todo profesor, sea principiante o experto, tiene creencias, ideas, metáforas, actitudes y hábitos de comportamiento que pueden orientar su acción e impedir, en algunos casos, su propio desarrollo profesional (Porlán 1989). Como se verá más adelante, la preparación de profesores no se puede resolver proporcionándoles instrucciones puntuales en forma de «recetas» a través de cursos ad hoc. Tanto la formación inicial como la permanente, en tanto que extremos de un mismo continuo, tequieren una profunda revisión que tenga en cuenta las adquisiciones de la investigación didáctica de las ciencias aunque sólo sea para no volver a caer en planteamientos actualmente ya superados. En el caso de la formación permanente se está comprobando que tanto aquellos planteamientos puntuales, supuestamente pragmátícos (Briscoe 1991), como aquellos otros más generales que prescinden de la materia a enseñar (Shulman 1992) no logran modificar la actividad de los profesores. En el caso de la formación inicial hay que cuestionar aquellos planteamientos que parten del supuesto de que la formación del profesorado es una yuxtaposición de saberes académicos que hay que aplicar en la práctica (McDermot 1990, Furió et al. 1992),

Figura 1

Esquema de necesidades formativas del profesor de ciencias.

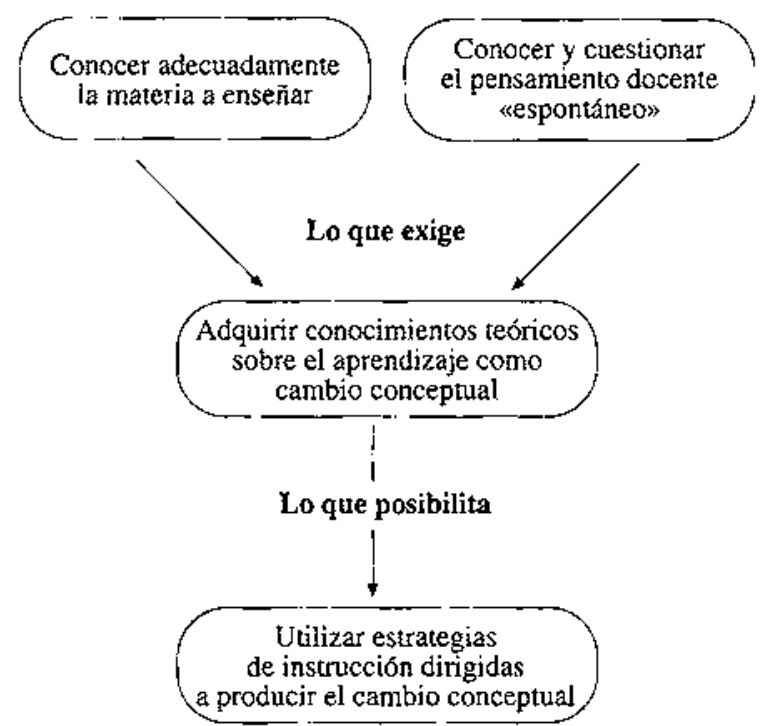

cuando su integración previa en la didáctica de las ciencias debería constituir un prerrequisito para poder ir solucionando una de las asignaturas pendientes bien conocida en esta formación inicial, como es la integra. ción de aquellos conocimientos teóricos con la preparación práctica a la docencia (Blackburn y Moisan 1986, Furió y Gill 1.989, Dumas-Carré et al. 1990).

La revisión de las tendencias actuales sobre la formación del profesoriado que se ofrece en este trabajo está de acuerdo con los resultados de la investigación realizada recientemente en este dominio y parte, en una primera aproximación, de una presentación sistémica de las ne. cesidades for mativas actuales de un profesor que quiera incorporar a sus estrategias didácticas aquellas exigencias derivadas de un planteamiento constructivista de la enseñanza. En este sentido el hilo conductor de este trabajo segu rá el esquema de la figura 1 donde se contemplan exigencias conocidas de antaño y que están siendo hoy revisadas por la investigación como es, por ejemplo, el conocimiento de la materia a enseñar, junto a otras más netamente constructivistas como pueden ser, por ejemplo, ia preparación de programas de actividades para la clase o la adquisición de habilidades para dirigir las actividades de los alumnos (Hewson y Hewson 1988 , Gil 1991, Furió 1992).

\section{UN PRIMER REOUISITO EN LA FOR- MACION IIEL PROFESORADO: CONOCER LA MATERIA A ENSENAR}

Incluso desdf: las primeras modelizaciones innatistas sobre la profissión en la primera mitad del siglo $\mathrm{xx}$, cuando se entendía que ser «buen profesor» era cuestión de vocación y de poseer ciertos dones, se argumentaba la necesidad de c ue aquél tuviera un buen conocimiento de la materia que había de enseñar a sus alumnos y alumnas. Esta idea sigue gozando de consenso general como se comprueba al preguntar a cualquier profesor de ciencias, y de facto -al menos, en nuestro país-es el único contenido real al qise se reduce en la práctica la formación inicial del profesorado de secundaria y de universidad (Furió y Gil 1989). Pero no siempre ha sido así desde la óptica de la formación del profesorado en general. Precisamente por reacción a aquel modelo artesanal de formación de frofesores basado en el conocimiento de la materia y en la adquisición de cierta experiencia al lado de un profesor experimentado (Zeichner 1987), surgieron propuestas ce formación que relativizaban la importancia dada a los contisnidos científicos incluso en la enseñanza secundaria. Una crítica reciente a estas propuestas la encontramos en las siguientes palabras de Shulman (1992):

... este paradigma (pedagógico) investigativo se centró exclusivament: en el comportamiento de los profesores[...] La prir.cipal cuestión era: ¿qué conductas distinguen a los profesores eficaces de los que no lo son? Ello significaba presuponer que habia caracteristicas generales de eficacia en la enseñanza que trascendía la materia, el nival de estudios y la mayor parte de otras componentes de la situación de enseñanza-aprendiza- 


\section{je[...] La invisibilidad de la materia persistió en lo que} yo empecé a llamar el paradigma perdido[...]

Coherentemente con esta idea de Shulman, la investigación en didáctica de las ciencias está demostrando que, precisamente, las insuficiencias de la preparación del profesor en los contenidos de la materia a enser̃ar es una primera dificultad que puede limitar gravemente el potencial innovador de cualquier profesor/a (Tobin y Espinet 1989). Cada vez más van apareciendo trabajos de formación inicial de profesores de ciencias que centran la atención en la exploración de sus conocimientos de la mate:ia. Así, por ejemplo, Gess-Newsome y Lederman (1993) ccmentan la importancia que tiene que los futuros profesores de biología se den cuenta no sólo de que hay que dominar los contenidos de la disciplina, sino también cle saber cómo los tienen trabados en lo que han denominado esquema conceptual de la disciplina ( subject matter structure, SMS); constructo que definen como las concepciones y/u organización del conocimiento de un área específica que tiene una persona. De acuerdo con las recomendaciones contenidas en el informe de 1990 de la National Research Council's Committee on High School Biology Education, estos mismos autores indican cue la posesión de un adecuado SMS -o mejor, ¡de varios!- ayudará al profesor en la selección de los tópicos más idóneos para ser incluidos en un currículo escolar dado.

Otro sintoma manifiesto de la importancia de conocer la materia Io constituye la inclusión habitual de componentes de actualización de contenidos en los programas de formación permanente. Por ejemplo, éste es el objetivo explícito del proyecto PSTS (Primary School Teachers and Science) descrito por Summers (1992) y que ha sido diseñado para favorecer la implementación de la actual reforma del National Curriculum que se está llevando a cabo en Inglaterra y País de Gales.

Ahora bien, La misma investigación en didáctica de las ciencias está llamando la atención sobre aquellos reduccionismos conceptuales que asimitan «conocer el contenido de la disciplina» con saber la estructura actual del tejido teórico propio de la disciplina, ya que dicho saber debe contener, además, conocimientos profesionales muy diversos que noestán contemplados en los estudios universitarios (Bromme 1988). Como muestra que puede ilustrar esta diversidad de significados y que, al propio tiempo, están sirviendo o pueden servir de marco a actuales of futuras investigaciones, se pueden citar brevemente las comentadas por Gil (1991) en un artículo anterior, tales como: a) saber los problemas y contextos que dieron lugar a la construcción de conocimientos científicos y, en particular, los obstáculos epistemológicos que se opusieron a dichos progresos, $b$ ) conocer las estrategias metodológicas empleadas en estas construcciones cicntíficas, c) conocer las interacciones existentes entre ciencia, técnica y sociedad asociadas a los conocimientos científicos, d) tener algún conocimiento de los desarrollos científicos recientes y sus perspectivas para poder adquirir una visión dinámica, no acabada, de la ciencia, $e$ ) saber seleccionar contenidos adecuados que den una visión correcta de la ciencia y, al propio tiempo, sean asequibles e interesantes a los estudiantes.
Entre estos saberes profesionales cabe destacar como necesidad formativa del profesorado de plena actualidad, su conocimiento de la historia y epistemología de la ciencia, asi como sus relaciones con la enseñanza (Saltiel y Viennot 1985, Gagliardi y Giordan 1986, Matthews 1990, Fillon 1991). En efecto, desde hace pocos años estamos asistiendo a una verdadera eclosión en este tipo de investigaciones con muy diversos síntomas que to ratifican. Así, por ejemplo, el núm. 1 del volumen 75 de Science Education está dedicado íntegramente a presentar una selección de los trabajos de la I International Conference on the History and Philosophy of Science and Science Teaching (Gruender y Tobin 1991). Una segunda edición de esta misma conferencia se celebró en mayo de 1992 en la Universidad de Ontario, en Kingston (Canadá). Por otra parte, ese mismo año vio la luz la revista Science and Education, dedicada explícitamente al estudio de las relaciones entre didáctica de las ciencias c historia y filosofía de la ciencia. Recordemos que, premonitoriamente, la revista Énseñanza de las Ciencias incluyó desde su nacimiento en 1983 una sección editorial destinada a trabajos sobre esta temática. Finalmente, y como dato consistente que corrobora la importancia que le está dando la comunidad científica al tema en la formación de profesores, se adjunta en el Cuadro 1 un extracto de las recomendaciones hechas por el grupo de Historia y Filosofía de la Ciencia en la V Reunión Latinoamericana de Educación en Física (V RELAEF 1993).

Todo ello viene a confirmar que hace falta mayor inves. tigación sobre la historia, la epistemología y la fillosofía de la ciencia y, a ser posible, contextualizada en la problemática de la didáctica de las ciencias (Meliado y Carracedo 1993). Precisamente la integración de este tipo de conocimientos históricos, sociales, etc. en el cuerpo teórico de la didáctica de la ciencia puede ayudar a fundamentar soluciones a cuestiones didácticas importantes como la mejora actitudinal del proceso de ense. ñanza-aprendizaje de las cjencias (Ajkenhead 1985, Solbes y Vilches 1989, Vilches, Acevedo 1993), la selección de contenidos de posibles diseños y desarrollos curricula-

\section{Cuadro I}

Resumen de las recomendaciones del grupo de historia y filosofia de la ciencia en la formación del profesorado de física en Latinoamérica (1993):

En relación con la formación de los profesores, la historia y filosofía de la ciencia es importante para propiciar:

a) Una mejor comprensión de la naturaleza del conocimiento científico.

b) Un mejor entendimicnto de los conceptos y tcorías de la Física.

c) Una comprensión de los obstáculos y posibles dificultades de los alumnos.

d) Una concepción de la ciencia como empresa colectiva $\mathfrak{c}$ histórica y el entendimiento de las relaciones con la técnica, la cultura y la sociedad. 
res en una situación concreta de enseñanza, etc. Al mismo tiempo, la adquisición de estos conocimientos será una herramienta vital para aquel profesor que moviéndose dentro de una orientación constructivista quiera «problematizar» sus enseñanzas y presentar una imagen de la ciencia más real, más contextualizada socialmente y menos «neutra». Por otra parte, la propia historia de la ciencia puede beneficiarse en alguna medida de las aportaciones realizadas por la investigación didáctica, pues las mismas dificultades epistemológicas de los estudiantes en la comprensión de los conceptos y teorías de la ciencia pueden señalar al historiador nuevas hipótesis interpretativas de los fenómenos que tal vez pudieran haberse dado en la comunidad científica pero que no han sido puestas de manifiesto en la historiografia (Bizzo 1993, Bullejos et al. 1993).

Pero no se puede reducir la formación del profesorado de ciencias al conocimiento de la materia, y por ello pasaremos a abordar un segundo aspecto que actualmente está destacando la investigación: el pensamiento docente espontáneo.

\section{UNA SEGUNDA LÍNEA DE INVESTIGACIÓN PROMETEDORA: CONOCERY CUESTIONAR EL PENSAMIENTO DOCENTE DE «SENTI- DO COMUN»}

Como es lógico en cualquier ciencia, los logros obtenidos por la investigación sobre las preconcepciones y actitudes en el aprendizaje de la ciencia han planteado nuevos problemas de profundizacion del conocimiento didáctico $y$, en consecuencia, han formulado nuevas preguntas como la siguiente: si se admite que las ideas previas de los/las estudiantes pueden facilitar u obstaculizar el anclaje de nuevos conocimientos científicos, ¿no es posible, de manera analógica, que los profesores de ciencias tengamos preconcepciones y actitudes respecto a la ciencia y la enseñanza que también puedan favorecer o impedir la adquisición de nuevos conocimientos di. dácticos? Paralelamente se vienen desarrollando en las ciencias de la educación, desde la segunda mitad de la década de los 80 , estudios centrados en el pensamiento y toma de decisiones del profesor (Calderhead 1986). En el dominio de la investigación en didáctica de las ciencias empieza a estudiarse que, amén del desconocimiento de la disciplina que se apuntaba en el apartado anterior, puede haber una segundo obstáculo importante que dificulte la implicación del profesor en actividades de imnovación que es lo que "ya sabe» acerca de la ensenanza. Se admite que el profesor posee saberes, creencias, comportamientos, etc. anteriores cuyo origen puede atribuirse al conocimiento inducido a través de su larga experiencia -primero, como alumno y después, como enseñante- y que han ido conformando de manera genérica una formación incidental (Carrascosa et al. 1985. Hewson y Hewson 1988, Porlán 1989). Estos conocimientos reciben distintos nombres, tales como pensamiento docente espontâneo, epistemología personal docente, preconcepciones de sentido común sobre la enseñanza, teorias implícitas, etc. Así, por ejemplo, en el trabajo de Tobin y Espinet (1989) citado anteriormente, se llega a
Ia conclusión de que esta formación incidental es una dificultad importante en la preparación del profesor para un cambio didáctico. Como bien indica Butts (Koballa et al. 1990), comentador invitado por Science Education en la revisión de la investigación didáctica hecha en 1988 , uno de los de:safíos clave en la formación del profesorado de ciencias es determinar qué conocimientos y creencias del protesor están relacionadas con su práctica y actitudes, cuín fuertes son estas relaciones y, finalmente, cuáles son las causas de las mismas. Una primera respuesta resper:to a preconcepciones docentes de «sentido común» ha sido ofrecida en un trabajo anterior por Gil (1991).

A continuación presentamos algunas conclusiones de trabajos de investigación que vienen a apoyar la existencia de estas preconcepciones docentes. Así, por ejemplo, Trumbull y Kerr (1991) abundan en la idea de que la principal infl tencia en el desarrollo profesional de los profesores es la forma en que han sido enseñados. Al entrevistar a varios científicos y profesores ayudantes universitarios para ver sus puntos de vista sobre la enseñanza, ésitos manifestaron que el fracaso de los estudiantes no podía considerarse por el profesor como un problema, puesto que se trataba de un whecho natural» o una «evidencia de sentido común». Coherentemente también mostraron gran escepticismo respecto a la posibilidad de investigar sobre la propia enseñanza.

Un caso particular de pensamiento docente espontáneo que está sienio exhaustivamente investigado en los últimos cuatrc años es el relativo a las creencias que tienen los profesores sobre la naturaleza de la ciencia y del trabajo científico, cómo éstas pueden afectar al currículo y, más en concreto, a la toma de decisiones del profesor en uni clase (Lederman y Zeidler 1987). A este respecto Duscill y Wright (1991) concluyen que esta toma de decisisnes del profesor está influenciada notablemente no sólo por el desconocimiento del contenido de Ia disciplina sino también por la falta de conocimiento sobre la naturileza y estructura de la ciencia. En el mismo sentido van las conclusiones del interesante artículo de Briscue (1991) donde se analizan las interacciones dinámicas existentes entre las creencias y metáforas utilizadas por un profesor y su práctica de enseñanza.

Respecto al cor tenido de estas creencias sobre la naturaleza de los conocimientos científicos que puede dar una imagen disiorsionada de estas construcciones, hay suficiente literatura que ataca tanto la visión excesivamente empirista-inductivista como la cientificista. Con relación a este último aspecto, conviene traer a colación los comentarios de Duschl (Koballa et al. 1990) abiertamente críticos con el autoritarismo imperante en la presentación de la ciencia en la educación primaria y secundaria donde se considera el conocimiento científico como absoltito y terminal. Este autor atribuye el origen de este cit'ntificismo subyacente en los profesores y científicos al positivismo lógico hegemónico en la primera mitad du este siglo Xx. Estas ideologias cientjficistas se caracterizan por su resistencia al criticismo, a no aceptar las conclusiones extraídas de la historia y sociología de la ciencia, ya que no cabe en sus esquemas de valores asumit cierta subjetividad en las propias 
construcciones científicas. De ahí la importancia que tiene defender diseños curriculares de una ciencia para todos donde no sólo se preste atención a los hechos científicos sino también se atienda a problemas humanísticos y sociales.

En relación con cuán fuertes son las preconcepciones empiristas en los diferentes colectivos de profesionales de la educación destaca el artículo de Pomeroy (1993) por sus paradójicas e interesantes conclusiones. Esta autora ha estudiado cuán fuertemente está arraigado el lógicoempirismo en científicos y profesores y qué implicaciones de las diferencias existentes entre ellos pueden fundamentar una mejora de la preparación del profesorado de ciencias. Entre sus conciusiones se apunta que los maes:ros son menos empiristas que los profesores de sccundatia y éstos, a su vez, lo son menos que los cientfficos. Ello se explica en base a que las nociones de los maestros sobre la naturaleza de la ciencia pueden venir de sus propios procesos de construcción del conocimiento pedagógico a través de sus experiencias y observaciones sobre cómo aprenden sus alumnos. En este supuesto, los programas de formación del profesorado deberían contemplar esta dinámica natural y construirse más sobre la práctica del profesor que en un entrenamiento específico sobre ciencia. Es decir, si el profesor aprende a ver que la práctica de la enseñanza puede relacionarse con la construcción de la ciencia misma (por cjemplo, hipotetizando que puede trabajar probando, evaluando y desarroliando teoría de la enseñanza y dei aprendizaje), cntonces podrá adquirir un punto de vista sobre la naturaleza de la ciencia desde la práctica personal de la enseñanza. Eso supone potenciar una integración de la teoría y la práctica que es totaimente consistente con los puntos de vista contemporáneos de la ciencia y con el creciente movimiento del profesor como investigador que desarrollaremos más adelante. Así pues, se necesita investigar en este sentido, diseñando programas de formación permanente del profesorado y explorando sus posibies efectos.

A título de ejemplo de investigación donde se ven las relaciones entre la epistemología docente de un profesor y su acción en la clase, se cita un trabajo reciente de Tobin et al. (1992). En esta comunicación se da cuenta de un estudio longitudinal donde se describen las dificultades de un profesor en cambiar sus enseñanzas debido a su episternología personal sobre la ciencia y el curículo. Este profesor tenía limitada experiencia en ciencias y pensatła que la ciencia a aorender consistía en un conjunto de verdades que tenía que ser aprendido por los estudiantes. Su práctica docente era consistente con cstas creencias. En efecto, organizaba la clase para que los estudiantes aprendicran mediante atención al profesor y lecturas personales; las actividades de laboratorio eran manipulativas y tenf́an por objeto aprender cl método cientifico; pedía a sus estudiantes que escribieran sus experiencias en informes normalizados con cabeceras estandars y cuya valoración se centraba en la adecuación del informe en la «forma correcta». Este comportamiento del profesor en cuestión era congruente con la interpretación de reglas que limitaban la acción de los estudiantes tanto en la forma de trabajar en el laboratorio como en la de presentación de los informes.
No obstante, hay trabajos de investigación similares como el de Hodson (1993) donde se han hecho entrevis. tas a profesores para ver si existe o no relación entre lo que se dice respecto a la naturaleza del trabajo científico y la manera de enfocar en sus clases tos trabajos de laboratorio. Al entrevistar a 12 profesores, el autor ha tipificado 4 clases de pensamiento sobre las construcciones científicas (inductivistas, verificacionistas, hipotéticodeductivistas y contextualistas) y ha mostrado claramente que existen, a veces, en el profesorado inconsistencias entre lo que se dice y lo que se hace. En un artículo muy reciente de Gil (1993) se han categorizado y descrito hasta diez visiones distorsionadas de la ciencia que pueden transmitirse en su enseñanza. También van apareciendo trabajos que muestran preconcepciones de los profesores sobre otros aspectos del proceso de enseñanzaaprendizaje como, por ejemplo, sobre la evaluacion (Alonso et al. 1991), etc.

En resumen, en este apartado hemos tratado de mostrar que se abre un período donde se va a desarrollar in extenso esta línea de investigación centrada en las idcas, comportamientos y actiłudes docentes de «sentido común» que, en general, son aceptadas de forma acrítica y que en una adecuada formación de profesores convendrá poner en cuestión en la medida que supongan algún obstácuio a su renovación. A tal efecto habrá que estudiar qué preconcepciones son y cuáles de éstas son más estables y consistentes aunque, en nuestra opinión, no deben ser muy fuertes atendiendo fundamentalmente a su origen incidental y a la brevedad, cuando no inexistencia, de la formación inicial. También habrá que disenar y experimentar estrategias de formación inicial y permanente que favorezcan un trabajo colectivo y que conduzca a los propios profesores en formación o en activo, a partir del análisis de sus propias concepciones, a ampliar sus recursos y a modificar sus perspectivas y, en definitiva, a orientar su propia formación como un cambio didáctico que tambien sea a la vez conceptual, metodológico y actitudinal pero aplicado a la enseñanza.

Ahora bien, este cambio didáctico del profesorado no puede ser concebido como una modificación puntual o de «parcheo» de estas preconcepciones docentes espontáneas como veremos ahora.

\section{LA FORMACIÓN DEL PROFESORADO DE CIENCIAS REOUIERE UN ESFUERZO DE FUNDAMENTACION TEORICA QUE INTEGRE TAMBIEN LAS NUEVAS EXI- GENCIAS PRACTICAS DE UN APRENDI- ZAJE CONSTRUCTIVISTA}

La necesidad de cambiar las concepciones y prácticas docentes espontáneas no debe llevarnos a buscar soluciones analíticas simples que supongan una preparación del profesorado excesivamente compartimentada en partes inconexas. Es fácil pensar que esta formación se subdivide, por una parte, en la adquisicion de saberes académicos que proporcionen los conocimientos de fa materia a enseñar y, por otra, en los relativos a las formas de enseñar en general. Esta idea ha sido fuertemente criti- 
cada por L. McDermott (1990) al referirse a los planes actuales de formación inicial del profesorado norteamericanos basados en la yuxtaposición de cursos de contenido impartidos por los departamentos de ciencias y cursos de educación específicos para esta preparación. En relación con los primeros, critica que se impartan con la misma metodología a todos los estudiantes indepen. dientemente de su especialización. Estas metodologías tienen como denominador común las siguientes caracte* rísticas:

a) La hegemonía de la extensión sobre la profundidad en el diseño del currículo, con amplios contenidos y muy poco tiempo para su desarrollo, que imposibilita una apropiación sígnificativa de los conceptos.

b) El formato expositivo de las clases, que estimula a la pasividad y a la recepción de conocimientos en los futuros profesores, en lugar de favorecer la actividad y la generación de los mismos.

c) La forma estandard de resolver los problemas mediante la repetición de algoritmos ya elaborados sin favorecer la creatividad ni las formas de razonamiento necesarias para abordar situaciones novedosas que, probablemente, serán las que puedan plantearse en su futura profesión.

d) El uso de material sofisticado en las prácticas de laboratorio -que, en general, no está disponible en los centros de secundaria- dirigidas a la verificación de conocimiento acabado que no ayuda a comprender la naturaleza de la actividad científica.

Respecto a los cursos de educación, esta autora comenta que se caracterizan por su típica descontextualización de los contenidos científicos, cuando de hecho los métodos a usar tienen mucho que ver con aquéllos. Como propuesta alternativa cita la necesidad de diseñar currículos específicos dirigidos a esta formación inicial del profesorado de ciencias de secundaria. Y si bien es necesario tener en cuenta la especificidad de estos estudios profesionales, hay que advertir que no suele ser condición suficiente para que los cursos típicamente científicos se den mejor, como se advierte fácilmente, en nuestro país, en los currículos de aquellas instituciones dedicadas precisamente a formar profesores de primaria en las especialidades de ciencias (Furió et al. 1992).

Por otra parte, es bastante difícil lograr esta transformación del pensamiento y comportamientos docentes de «sentido común» mediante acciones puntuales en forma de cursillos ad hoc. A este respecto hay experiencia suficiente sobre cómo se plantea, en general, la formación permanente del profesorado y cuáles son los resut. tados que obtiene. $Y$, aunque no hay mucha investiga* ción que haya evaluado seriamente los logros conseguidos por las administraciones educativas en el reciclaje del profesorado, hay una percepción general de que los cambios obtenidos en la práctica docente habitual son muy magros. Como indica Briscoe (1991) cada año son millares de profesores y profesoras los que participan en seminarios o asisten a cursos con la intención de perfeccionarse profesionalmente y cuando, de nuevo, llegan a sus clases creen que están mejor preparados para utilizar las nuevas orientaciones, Ios materiales renova* dos, etc. y, sin embargo, cuando menos se dan cuenta ya están actuan do como lo hacían antes. Ello no significa que aquellas ideas innovadoras no sean interesantes o que estas actividades de formación no hayan logrado sensibilizar ai profesorado. El problema esencial, a nuestro entender, está en que no se tiene en suficiente consideración -es más, ¡se suele menospreciar!- la enseñanza habitual como un sistema paradigmático de concepciones, creencias, comportamientos y actitudes generalmente inducidos de la experiencia y conocimientos anteriores que poseen cierta articulación y coherencia y que, como tai, da respuesta a la mayoría de los problemas que se plantean en a 1 aprendizaje de la ciencia. Desde este punto de vistá, se comprende que su transformación no sea sencilla y que su sustitución requiera no sólo un conocimiento claro de sus deficiencias a la luz de los resultados de la investigación didáctica, sino también tener elaboraço otro sistema alternativo, más articulado y coherente y que, al mismo tiempo, consiga mayor eficacia en la práctica docente. Ello significa rechazar una visión ecléctica y ateórica de la formación del profesorado y replantearla admitiendo sul carácter sisté. mico y paradi zmático, tanto si es para perfeccionar el modelo de ensiñanza-aprendizaje de transmisión verbal de conocimientos, como si es para sustituirlo por el nuevo paradigma constructivista. En nuestro caso, se apuesta por una formación del profesorado de ciencias basada en este paradigma emergente, pues entendemos que será capaz de dar respuesta a las dificultades del actual mediante un replanteamiento global que afecte a todos y cada urı de los aspectos điđácticos, así como a las posibles intərrelaciones entre ellos (Gil 1986).

En definitiva, el nuevo paradigma deberá contemplar la formación del profesorado como la construcción de un cuerpo teórico de conocimientos sobre el aprendizaje de Ias ciencias que, al propio tiempo, integre en el mismo las exigencias derivadas de una práctica docente de orientación constructivista. Pasemos, a continuación, a desarrollar un poco más extensamente en qué van a consistir estos dos aspectos y en qué medida la investigación actual los está ya teniendo en cuenta.

\section{Pero, ¿qué significa adquirir conocimientos teóricos sobre el aprendizaje de las ciencias?}

Esta exigencia de fundamentación teórica de la formación del profesorado por lo que se refiere a la adquisición de conocimiento; sobre el aprendizaje de las ciencias no debe significar una presentación desconectada de la propia materia a enseñar, ni mucho menos alejada de los problemas percitidos como tales por los profesores en activo o en formácición (Brincones et al. 1986). Se ha de plantear como una (re)construcción de conocimientos específicos integrados en un todo articulado en lo referente al aprendizaje de las ciencias (Viennot 1989). En particular, esta reconstrucción ha de partir de un diseño curricular y de un desarrollo que sean armónicos con las adquisiciones realizadas por la investigación didáctica $y$, en particular, con los resultados obtenidos en el aprendizaje de las ciencias de matiz constructivista. En cuanto a los conteridos de lo que puede significar «cons- 
truir conocimientos teóricos sobre el aprendizaje de las ciencias» se adjuntan las propuestas de Gil (1991) en el cuadro II.

\section{Cuadro II}

¿Qué conocimientos teóricos sobre el aprendizaje de las ciencias ha de contener un programa de formación de profesores? (Gil 1991)

a) Conocer la existencia de preconcepciones de los alumnos y alumnas sobre fenomenos naturales difíciles de reemplazar por los conocimientos científicos si no es mediante un cambio conceptuil y metodologico.

b) Saber que los/as alumnos/as aprenden significativamente construyendo conocimientos, lo que exige aproximar las actividades de aprendizaje de las ciencias (introducción de conceptos, diseño y realización de prácticas de laboratorio, resolución de problemas, etc.) a las características del trabajo científico.

c) Saber que los conocimientos son respuestas a cuestiones, lo que implica plantear el aprendizaje a partir de situaciones problemáticas de interés para los estudiantes.

d) Conocer el carácter social de la construcción de conocimientos cientifficos y saber organizar el aprendizaje consecuentemente.

e) Conocer la importancia que en el aprendizaje de las ciencias tienen el clima del aula y del centro así como las características personales del profesor (expectativas, compromiso personal con el progreso de los alumnos, etc.).

Este hilo conductor puede servir para la generación de programas coherentes en la formación del profesorado de ciencias como los expuestos en trabajos anteriores (Furió y Gil 1989, Gil et al. 1991).

Ahora bien, el desarrollo de uno de estos programas en clase debe tener, en palabras de Clark y Peterson (1986) expuestas en la tercera edición del Handbook of Research in Teaching, una conexión explícita-también podríamos decir coherencia metodológica- entre lo que piensá y dice el profesor de futuros profesores y cómo se desarrolla su acción en el aula. Por ejemplo, si se está defendiendo la necesidad de un currículo en la formación del profesorado de ciencias que forme un conjunto articulado y coherente, no se debe seleccionar una se. cuenciación lineal de capítulos estancos sin ningún nexo de uríón entre ellos. $O$, por ejemplo, si se considera importante contemplar aspectos motivacionales en uno de aquellos currículos, no se pueden dejar de lado las necesidades formativas sentidas como propias por los futuros profesores de ciencias en la planificación del programa. En este sentido, es interesante destacar como aportación teórica a la formación inicial del profesorado de ciencias, el contenido de un artículo reciente de Gunstone y otros (1993) donde se exponen los principios básicos que inspiran el programa de base constructivista que se está ensayando desde 1987 en la Universidad đe Monash (Australia) y que, en forma de proposiciones, se exponen sucintamente en el cuadro III.

Este mismo trabajo recoge también la importancia que los estudiantes de este programa dan a las principales tareas de una enseñanza constructivista, tales como: la selección del material didáctico, la adaptación del material seleccionado al nivel de los alumnos, la construcción de experiencia por los estudiantes al interaccionar con el contenido, la instrucción de los futuros profesores sobre las demandas y procedimientos de aprendizaje, la dirección del avance de los estudiantes y la necesidad de que el profesor de futuros profesores sirva a éstos como fuente de conocimientos y de destrezas. La mayor parte de estas proposiciones y tareas han de tenerse en cuenta también en la formación permanente de profesores en activo y, de hecho, se han estado aplicando paralelamente y sin conocerlas en algunos estudios que estamos haciendo sobre facilitación del cambio didáctico en profesores de ciencia en activo, bien en cursos de programas institucionales (Carrascosa et al. 1993) destinados a profesores con experiencia, bien en seminarios tutorizados para profesores con poco experiencia docente (Carnicer et al. 1993).

\section{Cuadro III}

Condiciones a tener en cuenta por los profesores que han de impartir conocimientos teóricos sobre el aprendizaje de las ciencias (Gunsto. ne et al. 1993).

a) Los futuros profesores y profesoras tienen necesidades que deben ser consideradas al planificar e implementar el programa de formación, así como los cambios que se producen en aquellas necesidades a lo largo del programa.

b) La transición de estudiante a profesor es fundamental y difícil y se ve facilitada cuando los futuros profesores trabajan estrechamente con sus colegas.

c) El profesor en formación es un estudiante que está activamente construyendo concepciones sobre la enseñanza y el aprendizaje basadas en sus experiencias personales y muy influenciadas por sus percepciones docentes anteriores al ingreso en el programa del curso.

d) Es más importante modelizar que simular las orientaciones de enseñanza-aprendizaje propuestas en el programa.

e) Los futuros profesores han de ver el trabajo programado en el curso como una experiencia educativa valiosa.

f) La formacion inicial es, por definición, insuficiente.

g) Las propuestas de Schön acerca del «profesional reflexivo» constituyen un modelo vital para quienes enseñan en el programa de formación inicial.

En resumen, hay que dar a la formación del profesorado una orientación y un contenido que vaya más allá del conocimiento de unos cuantos recursos y estilos de enseñanza (Gabel et al. 1987) y de la adquisición de habilidades puntuales, si realmente se desea lograr un cambio didáctico eficaz del pensamiento y de la acción docente de «sentido común». Para ello es fundamental reconocer el papel de la didáctica de las ciencias como núcleo vertebrador de los saberes académicos y la necesidad de que la investigación avance trabajos teóricos que favorezcan la construcción de este cuerpo específico de conocimientos capaz de integrar coherentemente sus resultados. Hacen falta, pues, estudios sobre la didáctica 
como ciencia de enseñar ciencias que determinen su núcleo conceptual duro en términos lakatosianos, sus anillos periféricos, etc. teniendo presente su carácter pluridisciplinar, sus diferentes programas de investiga. ción, sus colegios invisibles de investigadores, etc.

\section{La formación del profesorado ha de contemplar las exigencias de la práctica de una enseñanza constructivista}

Pero, no sólo los programas de formación de profesorado deben estar constituidos por núcleos integradores de los diferentes saberes académicos como componentes de una preparación teórica, sino que además han de ser capaces de integrar también aspectos más próximos a la acción educativa y a una iniciación en la práctica profe. sional del futuro profesor. Es decir, se ha de llegar incluso a modelizar métodos o estrategias de enseñanza a título de hipótesis de trabajo, a favorecer la vivencia de estos proyectos en el practicum y a evaluar de forma reflexiva y crítica las consecuencias de dichos tratamientos (Burbules y Linn 1991). A título de ejemplo de trabajos de investigación donde se están diseñando y experimentando programas de formación del profesorado de primaria y secundaria en ciencias en este sentido, tenemos el de la Universidad de Wisconsin-Madison (Hewson et al. 1992). Este programa de formación está basado en la integración de estrategias de aprendizaje de cambio conceptual con la investigación-acción y, según los autores, pretende erradicar las dos causas de fracaso que suelen tener estos currículos: $a$ ) la incapacidad para explorar y poner cn cuestión las preconcepciones que sobre la enseñanza traen los estudiantes a estos programas, y $b$ ) las insuficiencias para superar la separación «conocimiento-práctica» que normalmente se da en estos programas. Dos son sus componentes principales: a) un curso de métodos en la didáctica de las ciencias que trata de simular diferentes estrategias de cambio conceptual (trabajos en grupo, conferencias sobre constructivismo y preconcepciones de los estudiantes sobre fenómenos naturales, activida. des para discutir el contenido científico...), y $b$ ) una investigación-acción que comprende dos fases: una primera anterior al practicum donde se familiariza a los estudiantes con los constructos e hipótesis que se manejan en este tipo de estudios, al tiempo que empieza a planificar cada uno el proyecto de investigación-acción; y una segunda fase en que cada estudiante completa dicho proyecto, lo desarrolla en el practicum y sus resultados se discuten en un seminario del que forma parte junto a investigadores en este dominio.

Esta integración teoría-práctica puede tener soluciones diferentes según las posiciones constructivistas que se adopten respecto al aprendizaje, y, por tanto, Ias necesidades formativas del profesor diferirán de acuerdo con la enseñanza que se quiera llevar a la práctica. En el caso de que se propugne un aprendizaje de las ciencias como investigación, se habrán de plantear estrategias de enseñanza orientadas a que el cambio conceptual se produzca de manera gradual, no traumática y coherente con la naturaleza del trabajo científico. En este supuesto y de acuerdo con Wheatley (1991), la actividad habitual se focalizará hacia el tratamiento de situaciones problemá- ticas en las que el profesor adoptará, por una parte, el rol de facilitador del aprendizaje que permita la (re)construcción de conocimientos y, por otra, el de investigador en la acción de las situaciones concretas que se plantean en el aula. De acuerdo con ello, las componentes esenciales de esta enseñan:a por investigación pueden resumirse en:

a) Las tareas propias de la resolución de la situación problemática suponđrá saber preparar programas de actividades en la fase pre-activa (Driver y Oldham 1986), saber dirigir la actividad de los alumnos en este tipo de enseñanza y :aber analizar críticamente los resultados obtenidos en la fase post-activa;

b) Estos logros educativos se podrán debatir en el equipo docente en e. que se ha elaborado conjuntamente el programa de actividades y al qque pertenecerá el profesor en cuestión.

c) A su vez, istos equipos docentes, como grupos de investigación : innovación didáctica, podrán interaccionar con otros equipos de profesores a través de los medios ordinarios de comunicación e intercambio cientfficos (revistas esperializadas, congresos y simposios, etc.) contribuyendo asía la construcción social del conocimiento didáctico (Reyes 1991, Furió et al. 1993). Todo ello requerirá un trabajo del profesor cuyas principales tareas se han esquem atizado en la figura 2 y cuyo contenido se puede encontrar más detallado en el trabajo de Gil y Pessoa de Carvalho (1993).

Figura 2

Representación del trabajo del profesor y su contribución al conocimiento didáctico en la enseñanza por investigación.

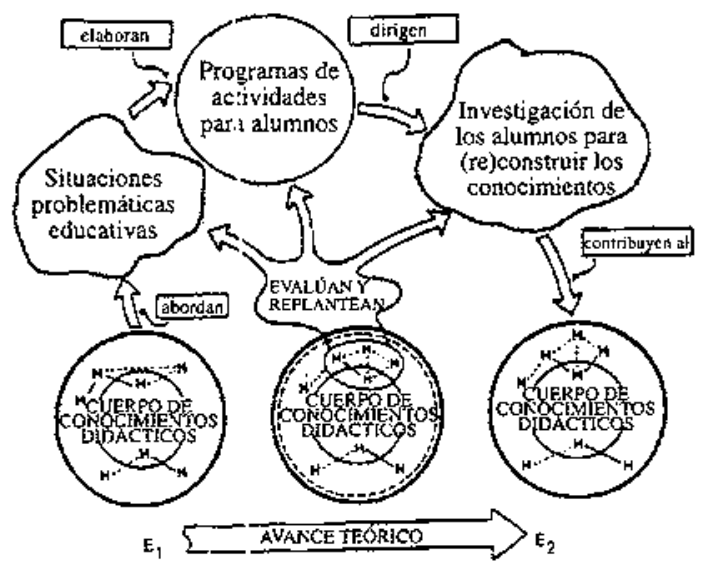

En definitiva, la formación actual de! profesorado no solamente debe vertebrar la construcción de saberes académicos relativos al conocimiento de la materia a enseñar con las formas y métodos propios de enseñarla, sino que además ha de integrar aquelios otros aspectos mås prácticos, inás próximos a la profesión, que se derivan de las nuevas necesidades formativas del profesor que ha de diriģir un aprendizaje de tipo constructivis- 
ta. A este respecto, se prevén, en un futuro próximo, investigaciones que tendrán como objeto el diseño y desarrollo de los contenidos de estos programas de formación de profesores, así como la evaluación de los mismos y de la actividad docente congruentes con el paradigma constructivista del proceso de enseñanzaaprendizaje de las ciencias.

\section{A MODO DE SÍNTESIS, OTRO PROGRAMA DE INVESTIGACION NO TAN UTÓPICO: LA INCORPORACIÓN DEL PROFESORADO A LA INVESTIGACION E INNOVACIÓN DIDÁCTICAS}

Pero, ¿no estaremos pidiendo a la figura del profesor de ciencias unas exigencias formativas exageradas? Tal vez. No obstante, creemos que hay que salir al paso de una preconcepción generalizada donde se asume que «enseñar es fácil» y que para esta tarea se requiere simplemente una apropiación individual del conocimiento didáctico en un corto período de tiempo y su posterior aplicación a la clase.

Cada vez es más patente que, tanto en la formación de futuros profesores como en el reciclaje de profesores en activo, hay que considerar la preparación a la investigación e innovación educativas como necesidad forma. tiva de primer orden. Pero esta preparación no debe entenderse como una componente a añadir a su forma* ción, sino más bien como una actitud que debe impregnar su actividad profesional cotidiana. Es decir, la inmersión del profesor en la investigación didáctica debería ser una exigencia de su propio trabajo, ya que el conocimiento de las principales líneas de investigación y de las implicaciones didácticas de sus resultados le proporcionarán orientaciones sin las cuales scrá bastante difícil fundamentar una crítica reflexiva de su práctica docente y, consecuentemente, también lo será la puesta a punto de innovaciones fructíferas que mejoren el aprendizaje (Furió 1984, Sáez 1987). Además si, por otra parte, el profesor tiene que asumir en clase el papel de director de una investigación (que ha de replicar) no sólo deberá conocerla sino que también deberá poseer vivencias en estas tareas investigativas y para ello requerirá la oportuna preparación teórico-práctica (Furió y Gil 1983).

Las denuncias y pronunciamientos de que la práctica docente y la investigación educativa han ido por caminos paralelos -cuando no divergentes- son bastante antiguos. Precisamente la investigación-acción que está hoy siendo revitalizada por los reconceptualistas de la formación del profesorado (Pinar 1989) es un ejemplo histórico de la necesidad de construir una «epistemología de: la práctica» que sirviera a la solución de los problemas percibidos como reales por la enseñanza, ya que la investigación educativa institucionalizada no los abordaba (Lewin 1946). Con posterioridad, estas denuncias se han hecho desde la propia investigación y pueden servir como ejemplos los esfuerzos de algunos autores para disminuir esta separación de la teoría y la práctica presentando los resultados de la investigaciốn de manera que puedan ser «consumidos» más fácilmente por el profesorado (Lawson et al. 1985) o la creación de pro- gramas como el de la NARST titulado Un profesor, $u$ investigador (Butzow y Gabel 1986).

En la actualidad no sólo se defiende que el profesorado se familiarice con el consumo de la investigación sino que sea el mismo el que la produzca. Puede hacerlo de forma colectiva en equipos docentes que preparan programas de actividades de aprendizaje, programas que, a título de hipótesis, experimentarán en clase y cuyos resultados serán motivo de análisis y reflexión que contribuirán al conocimiento didáctico y a su desarrollo profesional. No faltan, a este respecto, recomendaciones de congresos como el de formación de profesores iberoamericanos de física mencionado anteriormente donde se explicita que esta actitud investigadora debe impreg. nartodas las disciplinas de esta preparación. Como indican Kyle y otros (1991) al analizar el papel de la investigación en la enseñanza de las ciencias:

Una nueva imagen del rol del profesor está emergiendo. Además de poseer un conocimiento específico de la disciplina y un conocimiento de didáctica efectiva, los enseñantes deben disponer de tiempo para debatir ideas con sus colegas, participar en el desarrollo profesional $e$ investigar sobre la enseñanza y el aprendizaje.

A tal efecto, estos autores hacen una serie de recomendaciones respecto a la relación investigación-enseñanza de las ciencias que se han expuesto en el cuadro IV.

\section{Cuadro IV}

¿Cuál es el papel de la investigación en la enseñanza de las ciencias? (Kyle, Linn, Bitner. Mitchener y Perry 1991)

a) La investigación debería ser una empresa de colaboración entre maestros, profesores de EEMM y de universidad.

b) Todos los profesores deberfan ser investigadores en la acción.

c) La investigación debe estar próxima al aula.

d) Debería constituirse una asociación de investigadores

e) La investigación debería informar las decisiones políticas.

Así pues, se está fortaleciendo una tendencia a realizar investigaciones sobre formación de profesores que vayan en el sentido de imaginar estrategias donde se asocie indisolublemente docencia e investigación y que tengan como objetivo explícito el desarrollo de esta actitud investigadora en el profesorado. Los resultados que obtengan estas investigaciones habrá que tenerlos en cuenta para poder predecir qué condiciones se han de dar en aquella formación para mejorar la actitud y las expectativas de los futuros profesores y profesoras hacia la enseñanza. Condiciones sin las cuales creemos que difícilmente se pueda ilusionar a nuevas generaciones en la compleja pero rica y creativa tarea de enseñar ciencias. 


\section{NOTA}

* Versión actualizada de la conferencia que con este título se impartió en el IV Congreso Internacional sobre Investigación en la Didáctica de las Ciencias y de las Matemáticas, celebrado en Barcelona los días 13 al 16 de septiembre de 1993.

\section{REFERENCIAS BIBLIOGRÁFICAS}

ACEVEDO, J.A., 1993. Actitudes hacia el aprendizaje de tas ciencias físicas, naturales y matemáticas en BUP y COU. Un estudio sobre tres dimensiones, Enseñanza de las Ciencias, núm. extra, pp. 13-14.

AIKENHEAD, G.S., 1985. Collective decision making in the social context of science, Science Education, 69(4), pp. 453-475.

ALIBERAS, J., GUTIÉRREZ, R. y IZQUIERDO, M., 1989. La didáctica de les ciències: una empresa racional, Enseñanza de las Ciencias, 7(3), pp. 277-284

ALONSO, M., GIL, D. y MARTÍNEZ-TORREGROSA, I., 1992. Concepciones espontáneas de los profesores de ciencias sobre la evaluación, Revista de Enseñanza de la Física, 5 , núm. 2, pp. 18-38.

BAKER, D.R., 1991. A summary of research in Science Education1989, Science Education, 75(4), pp. 255-411.

BIZZO, N.M., 1993. Historia de la Ciencia y Enseñanza de la Ciencia: ¿Qué paralelismos cabe establecer?, Comunicación, Lenguaje y Educación, 18, pp. 5-14.

BLACKBURN, P. y MOISAN, C., 1986. The in-service training of teachers. (Presses Interuniversitaires Européennes: Maästrich)

BRINCONES, I., FUENTES, A., NIEDA, I., PALACIOS, M.J. y OTERO, J., 1986. Identificación de comportamientos y características descables del profesorado de ciencias experimentales del bachillerato, Enseñanza de las Ciencias, 4(3), pp. 209-222.

BRISCOE, C., 1991. The dynamic interactions among beliefs, role methaphores and teaching practices. A case study of teacher change, Science Education, 75(2), pp. 185-199.

BROMME, R., 1988. Conocimientos profesionales de los profesores, Enseñanza de las Ciencias, 6(1), pp. 19-29.

BULLEJOS, J., DE MANUEL, E. y FURIÓ. C., 1993. ¿Sustancias simples o elementos? Usos del término elemento químico en los jibros de texto, Enseñanza de las Ciencias, núm. extra, pp. 217-218.

BURBULES, N. y LINN, M., 1991. Science education and philosophy of science: congruence or contradiction?, International Journal of Science Education, 13(3), pp. 227-241.

BUTZOW, I.W. y GABEL, D., 1986. We all should be researchers, The Science Teacher, 53(1), pp. 34-37.

CALDERHEAD, J., 1986. La mejora de la práctica de la clase: aplicaciones de la investigación sobre la toma de decisiones en la formación oel profesorado, Actas del I Congreso Internacional sobre Pensamiento de los Profesores y Toma de Decisiones. (Servicio de Publicaciones de la Universidad de Sevilla).
CARNICER, J., FURIÓ, C., GARCÍA, M.J., MARTÍNEZ, R. MATOSES, V. y USÓ, F., 1993. Facilitando el cambio didáctico et. la formación permanente del profesorado de Ciencias. Estudio de casos, Enseñanza de las Ciencias, nưm. extra, pp. 4\}-42.

CARRASCOSA, J., et al. 1993. Los programas de formación permanente del profesorado de Física y Qufmica en la Comunidad Valenciana: un intento constructivista de formación didáctica, Enseñanza de las Ciencias, núm. extra, pp. 47-48.

CARRASCOSA, J., FURIÓ, C. y GIL, D., 1985. Formation du professorat, ies Sciences et changement methodologique. Vlèmes Journées Internationales sur l'enseignement Scientifique, pp. 301-308.

CLARK, C.M. y PETERSON, P.L., 1986. Teachers thought processes, en Wittrock, M.C. (ed.), Handbook of Research on Teaching (McMillan: Nueva York).

CRONIN-IONES, L.L., 1991. Science teaching beliefs and their influence on curriculum implementation: two cases studies, Journal of Research in Science Teaching, 38(3), pp. $235-250$

DRIVER, R., 1938. Un enfoque constructivista para el desarrollo del currículo en ciencias, Enseñanza de las Ciencias, 6(2), pp. 109-120.

DRIVER, R. y CILDHAM, V., I986. A constructivist approach to curriculum development in science, Studies in Science Education, 13, pp. 105-122.

DUMAS-CAREÉ, A., FURIÓ, C. y GARRET, R., 1990. Formación iricial del profesorado de ciencias en Francia, Inglaterra y Cales y España. Análisis de la organizacion de los estudios y nuevas tendencias, Enseñanza de las Ciencias, $8(3)$, pp. $274 \cdot 281$.

DUSCHL, R. y G.TOMER, D., 1991 . Epistemological perspectives on conceptua change: implications for educational practice, Journal of Ressearch in Science Teaching, 28(9), pp. 839858

FILLON, P., 1991. Histoire des sciences et reflexion épistemologiųue des élèves, Aster, 12, pp. 91-120.

FURIÓ, C., 1984 La renovació pedagògica i les Escoles d'Estiu, Butlletí dels Mestres, 190, pp. 2-5.

FURIÓ, C., 1992. ¿Por qué es importante la teoría para la práctica en la eơucación científica?, Aula de Innovación Educativa, 4-5, pp. 5-10.

FURIÓ, C. y GIL, D., 1983. Strategie pour mettre en liason formation des enseignants et recherche educative, Vèmes Journées Intemationales sur l'Enseignement Scientifique, pp. $409-416$.

FURIÓ, C. y GII, D., 1989. La dídáctica de las ciencias en la formación inicial del profesorado: una orientación y un 
programa teóricamente fundamentados, Enseñanza de las Ciencias, 7(3), pp. 257-265.

FURIÓ, $\therefore$, GIL, D., PESSOA DE CARVALHO, A.M. y SALCFDO, L.E., 1992. La formacion inicial del profesorado de Educación Secundaria: papel de las didácticas específicas, Investigación en la Escuela, 16, pp. 7-21.

FURIO, C., ITURBE, J. y REYES, J.V., 1993. Contribuciones a una propuesta constructivista de enseñanza-aprendizaje de las ciencias como investigacion, Enseñanza de las Ciencias, núm. extrá, pp. 59-60.

GABEL, et al., 1987. Science Education research interest of elementary teachers, Journal of Research in Science Teaching, 24(7), pp. 659-677.

GAGLIARDI, R, y GIORDAN, A., 1986. La historia de las ciencias: una herramienta para la enseñanza, Enseñanza de las Ciencias, 4(3), pp. 253-259

GESS-NE.WCOME, J. y LEDERMAN, N.G., 1993. Preservice Biology teachers' knowledge structures as a function of professional teacher education: a year long assessment, Science Education, $77(1)$, pp. 22-45.

GIL, D., 1983. Tres paradigmas básicos en la enseñanza de las ciencias, Enseñanza de las Ciencias, 1(1), pp. 26-33.

GIL, D. 1986. La metodología científica y la enseñanza de las cicncias: unas relaciones controvertidas, Enseñanza de las Ciencias, 4(2), pp. 111-121.

GIL, D., 1991. ¿Que han de saber y saber hacer los profesores de ciencias?, Enseñanza de las Ciencias, $9(1)$, pp. 69-77.

GIL, D., 1993. Contribución de la historia y filosofía de las ciencias a la transformación de la enseñanza de las ciencias, Enseñanza de las Ciencias, 11(2), pp. 197-202.

GIL, D., 1994. Diez años en la investigación de la enseñanza de las ciencias, Enseñanza de las Ciencias, 12(2), pp. 154-164.

GLL, D.. CARRASCOSA, J., FURIÓ, C. y MARTÍNEZTORKEGROSA, J., 1991 . la enseñanza de las ciencias en la educación secundaria. (Horsori-ICE: Barcelona).

GIL, D. y PESSOA DE CARVALHO, A.M., 1993. Formaçao de professores de Ciências. (Cortez Editora: Sao Paulo).

GIMENO, J., 1990. El perfeccionamiento como desarrollo de la profesionalidad docente, en Gil, D. (ed.), Formación de Formadores en Didáctica de las Ciencias, pp. 75-109. (Nau Llibres: Valencia).

GRUENDER, C.D. y TOBIN, K, 1991. Promise and prospect, Science Education, 75(1), pp. 1-8.

GUNSTONE, R.F., SLATTERY, M., BAIRD, J.R. y NORTHFIELD, I.R., 1993. A case study exploration of development in Preservice Science Teachers, Science Education, 77(1), pp. 47-73.

GUTIÉRREZ, R, 1987. La investigación en didáctica de las ciencias. Elementos para su comprensión, Bordón, 268, pp. 339-362.

HEWSON, P.W. y HEWSON, M.G., 1988. An appropriate conception of teaching science: a view from studies of science learning, Science Education, 72(5), pp. $597-614$
HEWSON, P.W., ZEICHNER, K.M., TABACHNICK, B.R., BLOMKER, K.B. y TOOLIN, R, 1992. A conceptual change approach to Science Teacher Education at the University of Wisconsin-Madison, en Proceedings of the Annual Meeting of the American Education Research Association (San Francisco CA).

HODSON, D., 1993. Philosophic stance of secondary school science teachers, curriculum experiences and children's understanding of science: some preliminary findings, Interchange, 24(1-2), pp. 41-52.

KYLE, W.C., LINN, M.C., BITNER, B.L., MITCHENER, C.P. y PERRY, B., I991. The role of research in Science Teaching: an NSTA theme paper, Science Education, 75(4), pp. 413-418.

KOBALLA, T.R., CRAWLEY, F.E. y SHRIGLEY, R.L.., 1990. A summary of research in Science Education 1988, Science Education, 74(3), pp. 253-407.

LAWSON, A.E., COSTENSON, K. y CISNEROS, R., 1986. A summary of research in Science Education, Science Education, 70(3), pp. 191-346.

LEDERMAN, N.G. y ZEIDLER, D.L., 1987. Science teachers' conceptions of the nature of science: do they really influence teaching behaviors?, Science Education, $71(5)$, pp. 721-734.

LEWIN, K, 1946. Action Research and minority problems, Journal of Social Issues, 2, pp. 34-46.

MATTHEWS, M.R., 1990. History, Philosophy and Science Teaching: A Rapprochement, Studies in Science Education, 18 , pp. 25-51.

MCDERMOTT, L.C., 1990. A perspective on teacher preparation in physics - other sciences: the need for special science courses for teachers, American Journal of Physics, 58(8), pp. 734-742.

MELLADO, V. y CARRACEDO, D., 1993. Contribuciones de la filosofía de la ciencia a la didáctica de las ciencias, Enseñanza de las Ciencias, 11(3), pp. 331-339.

NOVAK, J.D., 1988. Constructivismo humano: un consenso emergente, Enseñanza de las Ciencias, 6(3), pp. 213-223.

PINAR, W.F, 1989. A reconceptualization of Teacher Education, Journal of Teacher Education, enero-febrero, pp. 9-12.

PINTRICH,P.R., MARX, R.W. y BOYLE, R.A., 1993. Bcyond cold conceptual change: the role of motivational beliefs and classroom contextual factors in the process of conceptual change, Review of Educational Research, 63(2), pp. 167199.

POMEROY. D., 1993. Implications of tcachers' beliefs about the nature of science: comparison of the beliefs of scientists, secondary science teachers, and elementary teachers, Science Education, 77(3), pp. 261-278.

PORLÁN. R., 1989. Teoría del conocimiento, teoría de la enseñanza y desarrollo profesional: las concepciones epistemológicas de los profesores. Tesis doctoral no publicada. Universidad de Sevilla.

PORLÁN, R., 1993. Constructivismo y Escuela (Diada: Scvilla).

RESNICK, L.B., 1983. Mathematics and Science Learning: a new conception, Science, 220, pp. 477-478. 
RELAEF, V., 1993. Recomendaciones para la formación de profesores de física en Latinoamérica, Revista de Enseñanza de la Física, 6(1), pp. 33-46.

REYES, J.V., 1991. La resolución de problemas de Química como instrumento de cambio metodologico, Tesis doctoral no publicada. Universidad del Pais Vasco (Bilbao).

SÁEZ, M.J, 1987. La investigación-acción y la formación del profesorado, Investigación en la Escuela, 2, pp. 15-20.

SALTIEL, E. y VIENNOT, L., 1985. ¿Qué aprendemos de las semejanzas entre las ideas historicas y el razonamiento espontáneo de los estudiantes?, Enseñanza de las Ciencias, 3(2), pp. 137-144

SHULMAN, L.S., 1992. Renewing the pedagogy of teacher education: the impact of subject-specific conceptions of teaching, en Actas del Congreso "Las Didácticas Específicas en la Formación del Profesoradon, pp. 53-59. (Santiago de Compostela).

SOLBES, J. y VILCHES, A., 1989. Interacciones CTS: un instrumento de cambio actitudinal, Enseñanza de las Ciencias, $7(1)$, pp. 14-20.

SUMMERS, M., 1992. Improving primary school teachers' understanding of science concepts-theory into practice, International Journal of Science Education, 14(1), pp. 13-24.
TIBERGHIEN, A., 1985. Quelques eléments sur l'evolution de la recherche en didactique de la physique, Revue Française de Pédagogie, 72, pp. 71-86.

TOBIN, K. y ISSPINET, M., 1989. Impediments to change: applications of coaching in high school science teaching, Journal of Fesearch in Science Teaching, 26(2), pp. 105120.

TOBIN, K., TIPPINS, D. y HOOK, K., 1992. The long hard road from of,jectivism to constructivism, en Proceedings of the Second In'emational Conference on History and Philosophy of Science in Science Education. (Kingston: Canada).

WHEATLEY, C.H., 1991.Constructivist perspectives on Science and Mathemitics learning, Science Education, 75(1), pp. 921 .

VIENNOT, L., 1989. L'énseignement des sciences physiques object de recherche, Bulletin de l'Union des Physiciens, 716 , pp. $899-910$.

VILCHES, A., 1993. Las interacciones Ciencia-Técnica-Sociedad y la enseñanza de la Física y Química. Tesis doctoral no publicada. Unjuetsitat de València.

ZEICHNER, K.M., 1987. Preparing reflexive teachers. An overview of instructional strategies which have been employed in pre-service teacher education, International Journal of Educational Research, 11, pp. 565-575. 